\title{
Lidil
}

Revue de linguistique et de didactique des langues

$46 \mid 2012$

Typologie et description linguistiques

\section{Pour une description typologique des langues}

\section{Caroline Imbert et Nathalie Vallée}

\section{OpenEdition}

\section{Journals}

Édition électronique

URL : http://journals.openedition.org/lidil/3237

DOI : $10.4000 /$ lidil.3237

ISSN : $1960-6052$

\section{Éditeur}

UGA Éditions/Université Grenoble Alpes

\section{Édition imprimée}

Date de publication : 15 décembre 2012

Pagination : 5-21

ISBN : 978-2-84310-236-3

ISSN : $1146-6480$

Référence électronique

Caroline Imbert et Nathalie Vallée, «Pour une description typologique des langues », Lidil [En ligne], 46 | 2012, mis en ligne le 15 juin 2014, consulté le 22 septembre 2020. URL : http:// journals.openedition.org/lidil/3237 ; DOI : https://doi.org/10.4000/lidil.3237 


\title{
Pour une description typologique des langues
}

\author{
Caroline Imbert* ${ }^{*}$ et Nathalie Vallée $* *$
}

Ce numéro consacré à la linguistique typologique réunit sept contributions proposant chacune l'esquisse d'un phénomène linguistique dans une ou plusieurs langues, dans une approche fonctionnelle-typologique et typodescriptive des langues.

\section{Qu'est-ce que l'«approche fonctionnelle-typologique »?}

L'approche fonctionnelle-typologique est une approche non générative qui a été majoritairement appliquée aux domaines de la syntaxe et de la morphosyntaxe à la suite des travaux des West Coast Functionalists, tels que Tom Givón, Scott DeLancey, Colette Craig (alias Grinevald, contributrice de ce numéro), Charles Li, Sandra Thompson, dans les années 1970. Le format de cet article introductif ne permettant pas une présentation exhaustive, on pourra articuler cet exposé autour de cinq notions-clés, à notre sens essentielles pour comprendre cette approche : explication, fonction, motivation, dynamiques, facteurs externes.

\subsection{Explication}

C'est en réaction au transformationnalisme formel de Noam Chomsky que les fonctionnalistes de la Côte Ouest, parfois eux-mêmes issus de la mouvance chomskyenne (c'est le cas de Colette Craig, cf. Grinevald, ce volume) et surtout impliqués dans des terrains aux langues dites «exotiques», se sont littéralement accrochés aux travaux typologiques

* Caroline Imbert est MCF au Laboratoire LIDILEM (EA 609, Université de Grenoble).

** Nathalie Vallée est CR au Laboratoire GIPSA-Lab (UMR 5216, Université de Grenoble). 
de Joseph Greenberg pour les étoffer d'un cadre théorique, méthodologique et descriptif et donner au terme «typologie» une connotation bien spécifique à leur approche. La connotation «généraliste» du terme «typologie» renvoie évidemment aux notions de classification et de comparaison. En somme, la typologie consiste en une taxinomie linguistique visant à dégager des similitudes et des différences entre les langues.

Cependant, déjà à ce stade de la définition, l'approche fonctionnelle-typologique a mis en avant sa prise de distance avec le transformationnalisme ou pré-générativisme chomskyen. En effet, Tom Givón (2001-I : 20) s'interroge : si la typologie classe des structures linguistiques en types, puis rassemble ces types en méta-types, «en méta-types de quoi» est-on censé classer les types de structures? Il propose alors de classer les types de structures selon leurs fonctions (cf. 1.2) et s'oppose en cela à une conception purement structuraliste de la typologie.

Quant à la notion de comparaison, elle est au cœur du rôle théorique de la typologie : la comparaison translinguistique permet de découvrir des constructions et schémas qui ne sont visibles qu'à l'échelle translinguistique; elle permet de dégager des schémas typologiques, voire des tendances universelles dans les langues, que d'autres méthodes ne permettent pas de voir et d'appréhender. L'approche fonctionnelletypologique, sur ce point, s'est très tôt démarquée de l'approche initiale de Joseph Greenberg : si ce dernier se focalise sur l'uniformité des langues, les fonctionnels-typologues se sont rapidement intéressés à la variation dans les schémas translinguistiques ${ }^{1}$. En effet, qui dit variation, dit contrainte. Qui dit contrainte, dit possibilité d'identifier les facteurs reliant telle contrainte à telle variation, et donc possibilité de prévoir la présence de certains schémas dans les langues ainsi que leurs évolutions potentielles. Par ailleurs, des contraintes récurrentes sur des schémas de variation peuvent être motivées (cf. section 1.3) par des schémas cognitifs sous-jacents - ce point de convergence entre linguistique typologique et linguistique cognitive est essentiel pour les fonctionnels-typologues (cf. section 1.2).

1. William Croft (Croft, $2003: 47$, nous soulignons) parle ainsi de la distinction entre unrestricted universals et implicational universals : "Implicational universals capture a pattern in language variation, and differ from unrestricted universals, which account for uniformity, not variation. As such, implicational universals cannot even be discovered without cross-linguistic comparison.» 
Enfin, l'apport majeur de ces derniers à la notion de «typologie» est d'exploiter systématiquement sa valeur explicative et non uniquement descriptive. William Croft (2003:2) désigne même l'« explication » en typologie sous le nom de functional-typological explanation. Les fonctionnels-typologues pointent l'approche chomskyenne comme essentiellement descriptive malgré sa rigueur et sa vision «structuraliste» de la typologie (classer des types de structures en plus grands types de structures) : cette vision se résumerait finalement au simple «comptage» de la diversité des structures présentes dans les langues, sans jamais renvoyer aux possibles motivations fonctionnelles et cognitives sous-tendant cette diversité, et donc sans jamais tenter d'expliquer l'émergence et l'évolution (cf. 1.4) des structures linguistiques. Tom Givón (2001-I : 20) revient sur cette opposition entre les deux approches, qui aurait conduit des linguistes tels que Leonard Bloomfield (1933 : 19-20) à perdre de vue l'intérêt scientifique de construire une typologie, en sous-estimant l'importance, voire en niant la pertinence de la recherche même d'universaux linguistiques.

\subsection{Fonction}

L'approche fonctionnelle-typologique est fonctionnelle dans le sens où les schémas translinguistiques, les «types» qu'elle dégage, peuvent être articulés autour de domaines fonctionnels spécifiques, tels que ceux abordés dans ce volume : la Classification nominale (Noëllie Bon), la Valence verbale (Alice Vittrant, Camille Simon, Roser Gauchola), ou encore l'Espace (Marine Vuillermet). Un domaine fonctionnel est un ensemble de concepts (e.g. Trajectoire, Localisation, Figure, Fond) reliés à une fonction (e.g. expression de l'Espace); cette fonction répond à un besoin communicationnel du locuteur (e.g. se repérer et repérer des entités dans l'espace). Pour les fonctionnels-typologues, l'étude de la langue est ancrée dans les fonctions qu'elle assume et l'usage que le locuteur en fait. Ainsi, à l'instar de nombreuses approches récentes, ils préfèrent recueillir leurs données dans un registre oral et courant plutôt qu'écrit et soutenu. Plus spécifiquement cependant, leurs analyses soulignent l'articulation entre les niveaux phonologique, morphosyntaxique, sémantique, pragmatique et discursif, ainsi qu'entre les formes et les fonctions. Ces analyses se placent également en relation étroite avec la linguistique cognitive : elles reconnaissent que les fonctions qu'elles étudient dans la langue sont elles-mêmes sous-tendues par des processus cognitifs. En bref, la langue est un système de formes 
dont l'objectif est de transmettre des idées et chacun des éléments de ce système tend vers la réalisation de cet objectif.

\subsection{Motivation}

Cette fonction communicationnelle de la langue intègre la notion de motivation fonctionnelle (cf. DeLancey, 2001ms : 7). Dans l'approche fonctionnelle-typologique, l'étude de la langue trouve ses explications, pour un phénomène linguistique donné, dans l'étude des fonctions elles-mêmes mais aussi des processus diachroniques récurrents qui sont fonctionnellement motivés. La langue est donc appréhendée comme un produit de l'histoire, en constante évolution, et toute structure attestée en synchronie est le résultat d'une dynamique diachronique, elle-même motivée - et contrainte - par de nouvelles fonctions émergentes. En somme, les structures n'ont rien d'arbitraire : elles répondent à des règles mises en œuvre par des facteurs aussi bien internes qu'externes (les uns et les autres étant étroitement imbriqués). Tom Givón (2001-I : 25) remarque les fortes limitations pesant sur le nombre de types de structures possibles pour un domaine fonctionnel donné; il souligne alors la pertinence de l'étude des motivations et contraintes pour la linguistique typologique :

A serious theory of universals seeks to explain this seeming paucity of extant types. That is, it is honor-bound to ask: [...] What are the adaptive factors - be they cultural, communicative, developmental, cognitive, neurological or biological-that constrain diversity so severely? ${ }^{2}$

Le concept de motivation, intégré très tôt à leur approche par les fonctionnels-typologues, conduit les linguistes travaillant dans leur sillage à intégrer à leur démarche de description la prise en compte des dynamiques de systèmes et celle des facteurs externes.

2. «Une théorie des universaux digne de ce nom cherche à expliquer cette apparente pauvreté des types existants. De fait, on se doit de poser la question suivante : [...] Quels facteurs adaptatifs - qu'ils soient culturels, communicationnels, développementaux, cognitifs, neurologiques ou biologiques - contraignent si fortement la diversité [linguistique]?» (Traduction de Caroline Imbert.) 


\subsection{Dynamiques}

Une approche fonctionnelle-typologique n'a pas de sens si on la mène comme une entreprise strictement synchronique. L'étude de l'état synchronique de structures variées exprimant une même fonction ne permet pas d'en affiner la classification et encore moins d'en expliquer les mécanismes d'évolution. Pour paraphraser Tom Givón (2001-1 : 24), comprendre les structures sans comprendre les dynamiques d'évolution les ayant fait émerger n'a strictement aucun sens, car les structures sont ce qu'elles sont du fait de leurs adaptations successives à de nouvelles fonctions. Autrement dit, le lien existant entre structure et fonction réside précisément dans la notion d'évolution, c'est-à-dire de changement linguistique. L'approche fonctionnelle-typologique est donc de facto une approche panchronique (cf. Svorou, 1994 : 91), i.e. qui ne dissocie pas synchronie et diachronie.

Cependant, la notion de panchronie mise en avant par l'approche fonctionnelle-typologique ne se limite pas à une absence de distinction entre synchronie $v s$ diachronie (contrairement à ce que l'on peut parfois entendre, Ferdinand de Saussure et son structuralisme mettaient déjà en garde contre une distinction trop nette entre les deux et soulignaient l'apport de l'une à l'autre). Les fonctionnels-typologues appuient leur perspective panchronique sur deux notions essentielles : celle de (macro)grammaticalisation et celle de competing motivations (motivations en compétition).

Si la notion «classique» de grammaticalisation, issue à l'origine d'Antoine Meillet, implique simplement la tendance des éléments d'une langue au glissement du plus lexical au plus grammatical au cours de leur évolution, elle a été largement débattue et remodelée, et porte aujourd'hui beaucoup plus d'implications théoriques qu'à l'origine. Ces implications ont été largement relayées par l'approche fonctionnelle-typologique car elles font écho à plusieurs éléments théoriques fondateurs de cette approche. Christiane Marchello-Nizia (2006 : 16, nous soulignons), dans son ouvrage sur la théorie de la grammaticalisation, souligne notamment :

«[L'approche de la grammaticalisation] opère un décentrement de la réflexion traditionnelle, en focalisant son attention sur l'activité du locuteur en tant que telle, sur les processus cognitifs que l'usage du langage active chez lui [...]. L'homme en tant que locuteur est au centre de cette procédure d'analyse.» 
Ainsi, les schémas de grammaticalisation, en se focalisant sur les schémas d'évolution des structures, s'ancrent précisément dans le lien entre structure et fonction, et soulignent ainsi la prise en compte nécessaire des schémas cognitifs sous-jacents qui motivent ce lien dans toutes les langues humaines. Cette vision de la grammaticalisation est largement partagée au sein de l'approche fonctionnelle-typologique.

De façon plus cruciale, une telle vision permet d'étendre la notion de grammaticalisation à l'échelle de schémas, de systèmes linguistiques tout entiers (cf. Alice Vittrant dans ce volume sur les constructions à verbes sériels). Christiane Marchello-Nizia (2006 : 251) parle ainsi de «macro-grammaticalisation», i.e. «un changement structurel du macro-système grammatical, par une nouvelle hiérarchisation ou un nouveau découpage des unités de la grammaire [...]». La grammaticalisation peut donc affecter, au-delà des mots et constructions syntaxiques, jusqu'au «système de distinctions sur lequel se fonde une grammaire » (ibid.).

D'une part, la notion de macro-grammaticalisation intègre celle de gradience des catégories lexico-grammaticales, autrement dit la tendance courante des formes et constructions à ne pas pouvoir être classées dans une catégorie ou un type de construction avec certitude, à chevaucher plusieurs catégories ou type de construction en synchronie et à passer d'une catégorie ou d'un type à l'autre au cours de leur évolution (cf. notamment Rosch, 1978 ; Lakoff, 1987 ; Croft, 1991 ; Aarts, 2007).

D'autre part, la macro-grammaticalisation en tant que niveau d'analyse linguistique fait appel à une notion de «rééquilibrage» entre systèmes dans la langue :

- l'émergence ou la stabilisation d'un système peut être motivée par le déclin d'un autre système par un mécanisme de recyclage, comme clairement expliqué par Roger Lass (1990 : 80ff) :

[Languages] in their structure show a certain amount of bricolage; they are to some extent jury-rigged or cobbled together, and the remnants of old structures can be recobbled into new ones [...]. Languages may operate 'wastefully', dumping material that no longer does anything useful, or in a 'conservationist' mode, by recycling. This might in fact be a useful parameter for the typology of change. ${ }^{3}$

3. «[Les langues] montrent un certain niveau de bricolage dans leur structure; elles fonctionnent dans une certaine mesure par des assemblages et des com- 
- l'émergence ou la stabilisation d'un système peut provoquer le déclin d'un autre système par un jeu de compétition fonctionnelle et communicationnelle, favorable au mieux adapté, sous-entendu au système répondant le mieux aux besoins communicationnels des locuteurs - selon des critères pouvant être déterminés à la fois par des facteurs externes (e.g. économie, iconicité) ou internes à la langue (e.g. productivité morphosyntaxique, transparence sémantique, coût pragmatico-discursif).

Ces dynamiques de systèmes sont appelées competing motivations, notamment par John Du Bois (1985), et posent le problème de la coexistence de systèmes émergents, stabilisés et déclinants - question particulièrement étudiée par Caroline Imbert pour le domaine fonctionnel de l'Espace (cf. Imbert 2008, 2010, 2011) .

\subsection{Facteurs externes}

L'adaptation des structures au cours de leur évolution se fait en fonction de facteurs intralinguistiques, mais également extralinguistiques. Les sections précédentes ont permis d'évoquer plusieurs de ces derniers, notamment les schémas cognitifs qui constituent un point de convergence entre linguistique typologique et linguistique cognitive. Ces schémas n'ont pas été étudiés qu'en morphosyntaxe, mais également dans certains aspects de la phonologie - essentiellement le choix de ses unités (phonèmes, traits) et leur combinaison (syllabes, lemmes...) : ces derniers ont été étudiés en regard des processus de production et de perception de la parole, dans une démarche alliant la typologie des langues

binaisons de fortune, et les restes d'anciennes structures peuvent être recombinés en nouvelles structures [...]. Les langues peuvent pratiquer un certain "gaspillage", se débarrassant de matériau n'accomplissant plus rien d'utile, ou au contraire pratiquer un certain "conservatisme", en recyclant. Ce pourrait même être un paramètre utile à prendre en compte dans la typologie des changements linguistiques.» (Traduction de Caroline Imbert.)

4. Caroline Imbert argumente que l'un des défis descriptifs posés à l'approche fonctionnelle-typologique est de montrer en quoi ces dynamiques de systèmes sont un moteur essentiel de changements typologiques. Par ailleurs, qu'est-ce qui détermine le «succès» d'un système parmi plusieurs systèmes co-occurrents assumant la même fonction? Inversement, dans cet état de véritable «tension fonctionnelle», pourquoi certains systèmes déclinent-ils, laissant leurs fonctions à d'autres systèmes déjà stabilisés? 
et des systèmes à la modélisation des types (prédiction des systèmes), en passant par l'expérimentation en phonétique et en phonologie. Ces travaux s'inscrivent dans l'héritage des travaux en typologie de Philip Sedlak (1969) et des travaux en modélisation des années 1970 qui ont placé la distinctivité au centre de l'organisation des systèmes de sons de la parole - d'un point de vue articulatoire (Stevens, 1972), acoustique et/ou psycho-acoustique (Liljencrants et Lindblom, 1972). Plus précisément, ces travaux visent à démontrer les liens qui existent entre la phonologie des langues et les capacités sensori-motrices (cf. par exemple les travaux de De Boer, 2000; Demolin, 2007, 2008; Lindblom, 2000 ; Lindblom et al., 1984 ; MacNeilage \& Davis, 2000; Schwartz et al., 1997 ; Vallée et al., 2002; Vallée et al., 2009).

Enfin, il faut ajouter à ces facteurs externes celui de contact entre langues, sur lequel se focalise la linguistique aréale - elle aussi historiquement reliée à la linguistique typologique (cf. Noëllie Bon, ce volume). Outre les phénomènes d'emprunts et de création lexicale, une langue peut soumettre son système à divers processus de grammaticalisation et même subir un changement typologique progressif sur la base de facteurs qui ne sont pas, à l'origine, internes à son système linguistique, mais intégrés à ce dernier par contacts linguistiques.

\section{La notion de typodescription}

Du point de vue des méthodes de description, l'approche fonctionnelletypologique a été historiquement développée par des linguistes de terrain travaillant essentiellement sur des langues peu ou pas décrites.

Elle pose aujourd'hui les principes d'une nouvelle démarche de travail en linguistique typologique, celle de description typologique des langues. Cette stratégie descriptive, appelée ici «typodescription», prône un travail minutieux sur des données de première main et l'interaction possible et nécessaire entre cadre théorique, connaissances typologiques et description d'une langue. Colette Grinevald $(2005,2011)$ oppose ainsi à une vision «hiérarchique» de la théorie et de la description, une vision «horizontale», via une relation d'interdépendance entre ces deux aspects de l'étude linguistique. Ainsi, la théorie et la typologie ne doivent ni précéder ni suivre la description, mais en faire partie intégrante, dans une relation constante et horizontale de «va-etvient». Selon l'auteur (2005:2-3), une description de langue doit être le fruit d'un travail d'analyse «autant nourrie des connaissances des 
dernières avancées linguistiques typologiques qu'à l'origine de nouvelles avancées». Cette position, partagée par Denis Creissels dans ses travaux (cf. Creissels, 2006) mais également par Nicholas Evans \& Allan Dench (2006), ne va pas de soi pour tous les typologues. Colette Grinevald critique notamment une certaine tendance de ces derniers à extraire isolément les constructions d'une langue et à les comparer entre elles, sans tenir compte de leur place dans la grammaire, dans le système de la langue. Selon Colette Grinevald, cette place devrait au contraire être très importante, à la fois dans la description des langues et dans la construction d'une typologie. On verra ici une référence à peine voilée aux typologies «statistiques» à grande échelle comme celles du Surrey Morphology Group (SMG), du projet WALS (Haspelmath et al., 2005) ou comme la «typologie canonique» de Greville Corbett (2007), issue des travaux du SMG. Ces typologies manient souvent des centaines de langues à la fois; une démarche de typodescription élargira au contraire le panel de langues étudiées petit à petit, en prenant soin de répondre à l'exigence de ne manier que des données de première main - afin de maintenir l'ancrage de l'analyse dans l'usage (cf. sections 1.2 et 1.4). Mais Colette Grinevald se fait par ailleurs critique vis-à-vis des approches comparatistes, traditionnelles ou à visée typologique (notamment Lazard, 2001), qui, en voulant maintenir la pratique d'une comparaison translinguistique postérieure à la description, peuvent passer à côté de phénomènes linguistiques majeurs dans la langue au cours de sa description. Ainsi, d'une part, des connaissances typologiques préliminaires devraient élargir le spectre des constructions et schémas sous-jacents que le linguiste s'attend à trouver dans la langue qu'il décrit - ceci suppose donc que la typologie prenne en compte la place de ces constructions et schémas au sein des systèmes linguistiques. D'autre part, le processus de description d'une langue devrait en retour venir enrichir la typologie, et ce au cours de la description et en fonction des particularités internes au système de la langue. Cette démarche de «typodescription» constitue selon Colette Grinevald une démarche essentielle dans le cadre d'une approche moderne de la diversité des langues. En ouverture de ces réflexions et de manière intéressante dans le cadre de ce numéro de Lidil, l'auteur (2011: 48) souligne que l'interface même entre typologie et description peut être approchée de manière différente et être réalisée en interactions différentes, reconnaissant ainsi l'hétérogénéité de parcours et d'ancrage théoricodescriptif des linguistes maniant aujourd'hui cette interface : 
It would seem that doing typology takes many shapes today, linguists tending to espouse approaches and issues according to the kinds of relations they have to language data, to the types of languages they work with, and certainly according to both their linguistic training and personal preferences. ${ }^{5}$

\section{Pourquoi ce numéro?}

La «linguistique typologique» n'est pas une approche monolithique et, à ce titre, ce qu'elle implique comme outils théoriques, méthodologiques et descriptifs, ses qualités et ses limitations ne font pas l'objet d'un consensus parmi ses contributeurs, pas plus que chez ses détracteurs. Tel linguiste évoquera aussitôt Joseph Greenberg «le fondateur», ses travaux sur la phylogénétique des langues et ses classements de structures visant à dégager les fameux «universaux linguistiques », tandis qu'un autre formera le vœu qu'on ne limite pas le terme «typologie» à ces études certes pionnières mais essentiellement taxinomiques. Tel linguiste évoquera en France les travaux de Gilbert Lazard, ses travaux sur l'actance où la notion de «fonction» trouve une place plus centrale que celle de structure, tandis qu'un autre pointera la persistance d'une utilisation de la typologie comme outil de comparaison et non de description, ce qui aboutirait encore à une approche taxinomique, celle des fonctions plutôt que des structures. Tel linguiste évoquera les West Coast functionalists (notamment Tom Givón, Scott DeLancey, Colette Craig, Sandra Thomson, Charles Li), «les héritiers détracteurs » du structuralisme et du distributionnalisme, pour qui la fonction est tout, la synchronie rien sans la diachronie et la langue un organisme s'adaptant à un environnement, tandis qu'un autre regrettera l'abandon du formalisme et de sa rigueur par pur rejet de Noam Chomsky et de son approche transformationnelle d'alors.

Ces trois grandes tendances dans nombre de discussions autour de l'histoire et de la «valeur» de la linguistique typologique ne sont pas les seules et toutes les ramifications de cette dernière ne pourraient être

5. «Il semble que faire de la typologie peut prendre plusieurs formes aujourd'hui, les linguistes ayant tendance à adopter approches et problématiques selon la relation qu'ils entretiennent avec les données, les types de langues sur lesquels ils travaillent, et certainement selon leur formation linguistique et leurs préférences personnelles.» (Traduction de Caroline Imbert.) 
évoquées dans la présente introduction. On peut cependant noter un point récurrent de rassemblement ou de discorde parmi ces tendances, celui de la frontière entre le comparatiste, le contrastif et le typologique. Au plus chaud des débats, la visée comparatiste sera immanquablement taxée d'archaïsme et de «monomanie phylogénétique» en référence à la grammaire comparée et à ses nombreuses études sur l'indo-européen; la visée contrastive fera l'objet d'une mise à part expéditive pour son manque d'ambition en ne sélectionnant que trop peu de langues et en se projetant plus dans l'appliqué que dans le fondamental; la visée typologique sera âprement critiquée pour sa dispersion tout aussi extrême et sa posture arrogante d'approche post-moderne n'ayant rien inventé.

On peut sentir poindre dans toutes ces discussions en France et à l'étranger, quelle que soit la visée et l'école théorique embrassée, la question de la place, de la productivité et de la pertinence du simple fait de mettre en perspective plusieurs langues, familles ou groupes de langues : cette mise en perspective doit-elle précéder ou suivre la description? Est-elle annexe ou nécessaire? Qu'apporte-t-elle à la linguistique descriptive? Or, c'est en France que depuis une vingtaine d'années se construit et s'étoffe une vision de la description où théorie, typologie et description proprement dite ne s'inscrivent plus dans une relation «de pré- et de post-», dans une relation hiérarchique ou l'une gouvernerait l'autre. Cette approche «typodescriptive», introduite plus haut, a été ardemment défendue et argumentée dans le troisième volume (2011) des Cahiers de Faits de Langues par Colette Grinevald, laquelle ouvre le présent numéro de Lidil.

C'est la vocation de ce numéro de prêter un auditoire varié (descriptivistes, didacticiens, théoriciens, acquisitionnistes, dont ceux du FLE) à cette approche bâtie par des linguistes de terrain engagés aussi bien dans la description linguistique que la documentation des langues. Mais c'est aussi sa vocation de prêter la voix aux héritages variés de ces typodescriptivistes, que l'on verra transparaitre dans leurs contributions.

Ces dernières forment une palette d'esquisses, de l'Amérique du Sud à l'Asie en passant par l'Europe et la zone occitane, où chaque linguiste nous parle de sa langue de terrain, de ses données et de ses choix d'approche pour les éclairer. Il faut souligner la présence particulièrement appuyée dans ce numéro de jeunes linguistes : il était important pour nous, éditrices de ce numéro, de mêler les voix de chercheurs chevronnés à celles de doctorants et jeunes post-doctorants. Notre appel à contribution encourageait ces derniers à nous faire part de leurs travaux, de leur point de vue sur leurs héritages à la fois théorique et descriptif, et 
aussi bien de leurs certitudes que de leurs interrogations quant à l'apport de la typologie dans leur démarche de description linguistique.

\section{Présentation des contributions}

Colette Grinevald ouvre ce dossier par ses réflexions sur l'interface typologie-description et les interactions qu'elles entretiennent au cours de la production d'une «typologie évolutive» (working typology, cf. Grinevald, 2011) des systèmes linguistiques. La typologie évolutive s'inscrit dans une démarche de typodescription ancrée dans une méthodologie de terrain. Colette Grinevald défend l'idée que, pour les linguistes s'engageant à la fois dans une mission scientifique de description et dans une mission socioculturelle de documentation, le rapport aux données entraine souvent et appelle la nécessité de cette interaction constante et «horizontale» entre typologie et description linguistiques. Pour appuyer son propos, Colette Grinevald offre à ce numéro la synthèse inédite d'une partie de ses travaux de chercheur, en nous montrant comment, au cours de sa carrière, elle a mené l'élaboration d'une typologie de la Classification nominale. À partir de données du jakaltek popti', langue maya du Guatemala et terrain d'origine de l'auteur, cette dernière a dû amender et étoffer sa typologie au fil des défis posés par d'autres systèmes amazoniens et australiens, et, plus récemment, par un système de classificateurs en égyptien hiéroglyphique. Enfin, l'article ouvre l'application de la typologie évolutive à d'autres domaines fonctionnels, comme l'expression de l'Espace et de la Trajectoire, et propose des perspectives pour la recherche linguistique future.

En droite ligne de l'approche proposée par Colette Grinevald, Nö̈llie Bon aborde le système de Classification nominale en stieng, langue môn-khmer du Cambodge, dans une perspective fonctionnelletypologique et aréale. L'auteur concentre son analyse sur un système de «classificateurs numéraux de tri», en propose une description tout en les mettant en perspective avec l'ensemble du système de Classification nominale de la langue. En partant de ce que la typologie des classificateurs nous enseigne sur ce type de systèmes, Noëllie Bon s'applique tout d'abord à distinguer les différents sous-systèmes observés en stieng (noms de classe vs classificateurs, puis classificateurs mensuraux vs classificateurs de tri), puis propose une description fonctionnelle, morphosyntaxique, sémantique et pragmatico-discursive des classificateurs numéraux de tri, toujours en les faisant contraster avec les autres sous- 
systèmes. La description sémantique est particulièrement étoffée, avec une évaluation précise des différents critères entrant dans la différenciation fonctionnelle entre éléments au sein de l'inventaire des classificateurs. Il s'agit d'une description nouvelle d'un système non encore décrit dans la littérature, dont l'apport à la typologie et aux langues môn-khmer est d'autant plus intéressant qu'il s'agit d'une description dynamique articulée autour d'un paramètre d'analyse fondamental des systèmes de Classification nominale, celui de grammaticalisation et plus particulièrement de macro-grammaticalisation.

Marine Vuillermet se concentre sur le domaine fonctionnel de l'Espace et propose une analyse fine de l'expression du «mouvement associé» en ese ejja. L'expression du mouvement associé est un phénomène originellement décrit dans les langues australiennes puis identifié dans un certain nombre de langues amérindiennes, notamment en Amazonie dans des langues de la famille takana, dont fait partie l'ese ejja. Le mouvement associé appartient au paradigme verbal et permet d'associer un mouvement à l'action décrite dans le Procès. Pour reprendre les mots de l'auteur, il inscrit «l'action dans un contexte spatial de la même manière que les marques de temps situent l'action dans un contexte temporel». Marine Vuillermet nous montre son cheminement à travers les données de sa langue de terrain et démontre au fil de ce cheminement l'intérêt typodescriptif de ses données : il s'agit d'une part d'enrichir la typologie du mouvement associé, encore trop peu pourvue, et d'autre part d'affiner l'identification du mouvement associé, souvent confondu dans d'autres langues amérindiennes avec des systèmes de type directionnels ou motion-cum-purpose. Ainsi, si la typologie a permis à l'auteur d'identifier ce phénomène en ese ejja, l'ese ejja vient en retour amender la typologie et donner une base descriptive plus solide à l'identification du mouvement associé à l'échelle translinguistique.

L'apport de l'analyse typologique dans la description de constructions linguistiques complexes et de leur fonctionnement est clairement démontré par Alice Vittrant dans son étude novatrice des constructions à verbes sériels (CVS) du birman. Les CVS font actuellement l'objet de nombreuses discussions quant à leur définition et leur caractérisation, la notion de sérialisation verbale ne renvoyant pas à un phénomène monolithique. L'analyse proposée par Vittrant met en évidence, à partir d'un ensemble de constructions sérielles, que des formes de surface identiques peuvent correspondre à des structures sous-jacentes différentes. Ainsi, elle met en lumière quatre types de CVS en birman et propose 
de les placer sur un continuum lexicalisation-grammaticalisation, confirmé par des études diachroniques sur les processus de sérialisation.

Concernant également la morphosyntaxe verbale, l'article de Camille Simon confronte et combine les enseignements de Lazard, Givón et Creissels sur la Valence et la voix. Ceci la conduit à remettre en cause certaines bases de la description des systèmes de Valence verbale, en montrant que l'examen de la variation de la Valence sémantique plutôt que syntaxique est pertinente pour définir un changement de la structure actancielle du verbe en tibétain. Sa démonstration s'appuie sur l'implication ou non par le verbe de la présence d'un Participant dans l'énoncé ou dans le contexte, en testant le changement ou non de Valence sémantique à partir de la réaction d'un locuteur natif lors de l'écoute de ces énoncés. Le critère de la Valence sémantique est d'abord appliqué pour distinguer les actants centraux et les actants périphériques, puis pour analyser la réalisation morphosyntaxique des actants et leur rôle syntaxique. Cette démarche permet à Camille Simon de montrer l'existence de deux types de diathèse en tibétain (réorganisation des rôles syntaxiques et sémantiques non marquée par un morphème verbal de voix) et de considérer d'autres processus de marquage casuel des actants qui ne fonctionnent pas comme un mécanisme de voix.

Dans une démarche mêlant une inspiration clairement contrastive à une perspective fonctionnelle-typologique, Roser Gauchola revisite la voix passive en français et en espagnol. La voix passive est ici considérée selon la fonction de Participation qui situe le rapport entre le Participé (le verbe) et les Participants (syntagmes nominaux). La passivation y est décrite comme action prototypique caractérisable par des traits relevant des fonctions syntaxique, sémantique et communicative. L'analyse contrastive des techniques passivantes dans les deux langues est déclinée par l'auteur en deux types : les constructions périphrastiques et les constructions réflexives. L'approche fonctionnelle permet à Roser Gauchola de décrire à la fois les variations morphologiques et celles du schéma actanciel, en démontrant l'importance du flux attentionnel des locuteurs dans les différents types de construction. L'analyse de Roser Gauchola s'ancre donc dans plusieurs niveaux d'analyse étroitement imbriqués, la morphologie, la syntaxe, la sémantique et l'interface pragmatico-discursive.

Lucie Amaro clôt ce volume par une esquisse phonologique et dialectologique d'une variété occitane (le parler alpin d'Usseaux). Elle se concentre, dans cette variété, sur les «polyévolutions» phonolo- 
giques attestées du L intervocalique latin (conservation, rhotacisme, vocalisation) en s'appuyant aussi sur d'autres données synchroniques et diachroniques de l'espace occitan. Tout en maintenant un ancrage fort dans la tradition descriptive de son terrain (perspective résolument dialectologique, apport de données historiques et comparatives au sein de l'aire dialectale), Lucie Amaro tente d'une part une incursion dans ce que l'on sait d'autres variétés linguistiques romanes (notamment portugais, rhéto-roman, français, espagnol, italien) et pousse d'autre part l'exploration vers d'autres groupes indo-européens tels que le germanique (variations dialectales de l'anglais) et le grec. Ce positionnement, à la fois dans la dialectologie romane et au seuil des différentes ouvertures possibles vers la typologie, pose in fine la question de l'intégration d'une visée typodescriptive à une telle étude : quel est l'apport de cette intégration et surtout comment doit-elle se faire lorsque, à défaut d'avoir constitué d'emblée une démarche descriptive dans l'analyse, cette dernière l'incorpore au fil de son développement?

\section{RÉFÉRENCES BIBLIOGRAPHIQUES}

AARTS B. (2007) : Syntactic gradience: The nature of grammatical indeterminacy, Oxford, Oxford University Press.

Bloomfield L. (1933) : Language, New York, Henry Holt and Co.

CORBETT G. (2007) : «Canonical typology, suppletion and possible words», Language, 83, 8-42.

CReISSELS D. (2006) : Syntaxe : une introduction typologique, Paris, Hermès. Croft W. (1991): Syntactic Categories and Grammatical Relations: The Cognitive Organization of Information, Chicago, University of Chicago Press.

CROFT W. (2003) : Typology and Universals, Cambridge (MA), Cambridge University Press.

De Boer B. (2000) : «Self-organization in vowel systems », Journal of phonetics, 28, 441-465.

DeLancey S. (2001ms) : Lectures on Functional Syntax. Disponible sur $<$ http://pages.uoregon.edu/delancey/sb/fs.html>.

Demolin D. (2007) : «Phonological Universals and the control and regulation of speech production», dans M-J. Solé, M. Ohala et P. Beddor (dir.), Experimental approaches to phonology, Oxford, Oxford University Press, 75-92.

Demolin D. (2008) : «The Frame/content Theory and the emergence of consonants», dans K. Zjado et B. Davis (dir.), The syllable in Speech Production, Oxford, Laurence Erlbaum, 93-110. 
Du BoIs J. (1985) : «Competing motivations », dans J. Haiman (dir.), Iconicity in syntax, Amsterdam, John Benjamins, 343-366.

Evans N. et Dench A. (2006) : «Introduction: Catching Language», dans F. K. Ameka, A. Dench et N. Evans (dir.), Catching Language: The Standing Challenge of Grammar Writing, Berlin/New York, Mouton de Gruyter, 1-40.

Givón T. (2001) : Syntax: An introduction, Amsterdam, John Benjamins.

Grinevald C. (2005) : «De la typologie contextualisée», La typologie en France aujourd'hui, $7^{\mathrm{e}}$ Journée scientifique de la Cellule de Recherche en Linguistique (CRL), Université de Paris 7, 28 mai 2005.

GRINEVALD C. (2011) : «On constructing a working typology of the expression of path», Faits de Langues : les Cahiers, 3, Paris, Ophrys.

Haspelmath M., Dryer M. S., Gil D. et Comrie B. (dir.) (2005) : The World Atlas of Language Structures, Oxford, Oxford University Press. Disponible sur <http://wals.info/>.

IMBert C. (2008) : Systems dynamics and functional motivations in Path coding. A typological description of Homeric Greek and Old English, thèse de doctorat, CNRS / Université Lyon 2, disponible en ligne.

ImBert C. (2010) : «Multiple preverbation in Homeric Greek: A typological insight», CogniTextes, 4. Disponible sur <http://cognitextes.revues. $\operatorname{org} / 387>$.

Imbert C., Grinevald C. et Söres A. (2011) : «La catégorie de "satellite" de Trajectoire : pour une approche fonctionnelle-typologique», Faits de Langues : les Cahiers, 3, Paris, Ophrys.

LAKOFF G. (1987) : «Cognitive models and prototype theory», dans U. Neisser (dir.), Concepts and conceptual development, Cambridge, Cambridge University Press.

Lass R. (1990) : «How to do things with junk: exaptation in language evolution », Journal of Linguistics, 26, 79-102.

LAZARD G. (2001) : Études de linguistique générale : typologie grammaticale, Leuven-Paris, Peeters.

LiLjencrants J. et LindBlom B. (1972) : «Numerical simulation of vowel quality systems: The role of perceptual contrast», Language, 48, 839862 .

LindBlom B. (2000) : «The interplay between phonetic emergents and the evolutionary adaptations of sound patterns », Phonetica, 57, 297-314.

Lindblom B., MacNeilage P. F. et Studdert-Kennedy M. (1984) : «Selforganizing processes and the explanation of languages universals », dans B. Butterworth, B. Comrie et O. Dahl (dir.), Explanation of Language Universals, The Hague, Mouton de Gruyter, 181-203.

Marchello-Nizia C. (2006) : Grammaticalisation et changement linguistique, Louvain, Duculot. 
MacNeilage P. F. et Davis B. L. (2000) : «On the Origin of Internal Structure of Word Forms », Sciences, 288, 527-531.

Rosch E. (1978) : «Principles of categorization», dans E. Rosch and B. B. Lloyd (dir.), Cognition and categorization, Hillsdale (NJ), Erlbaum, 27-48.

Schwartz J.-L., Bö̈ L.-J., Vallée N. et Abry C. (1997) : «The Dispersion-Focalization Theory of Vowel Systems», Journal of Phonetics, 25, 255-286.

SEDLAK P. (1969) : «Typological considerations of vowel quality systems», Working Papers on Language Universals, Stanford University, 1, 1-40.

STEvens K. (1972) : «The Quantal Nature of Speech: Evidence from Articulatory-Acoustic Data», dans P. B. Denes (dir.), Human Communication: a Unified View, New York, McGraw-Hill, 51-66.

Svorou S. (1994) : The Grammar of Space, Typological Studies in Language, 25, Amsterdam, John Benjamins.

Vallée N., Bö̈ L. J., Schwartz J. L., Badin P. et Abry C. (2002) : The weight of substance in phonological structure tendencies of the world's languages, Berlin, ZAS Papers in Linguistics, 28, 145-168.

Vallée N., Rossato S. et Rousset I. (2009) : «Favoured syllabic patterns in the world's languages and sensorimotor constraints », dans F. Pellegrino, E. Marsico, I. Chitoran et C. Coupé (dir.), Approaches to phonological Complexity, Berlin, Mouton de Gruyter, 11-39. 